\title{
El estudio de la significancia y los indicadores de riesgo antrópicos para la preservación de colecciones de la memoria trágica en una exposición temporal
}

\author{
Ana Galán-Pérez, Eduarda Vieira
}

\begin{abstract}
Resumen: Las colecciones procedentes de los hechos históricos en un contexto bélico como fueron los campos de concentración nazi de la II Guerra Mundial, poseen un valor cultural y social relevante como testimonio material de los hechos. Conseguir el equilibrio a partes iguales entre su accesibilidad social y su conservación es el principal reto al que se enfrentan los museos memoriales como el Museo Estatal de Auschwitz-Birkenau, al que hay que sumar todos los riesgos añadidos que suponen su cesión en una exposición temporal, especialmente aquellos riesgos con origen antrópico, sean fortuitos o intencionados. El análisis crítico de estos indicadores nos ayudará a reconocer las posibles emergencias y consiguientes daños en las colecciones de la memoria en tránsito y abordar su conservación desde la perspectiva de la sostenibilidad.
\end{abstract}

Palabras clave: colecciones de memoria trágica, riesgo antrópico, exposición temporal, sostenibilidad, Museo Estatal de AuschwitzBirkenau

\section{The study of the significance and anthropic risk indicators for the preservation of collections of tragic memory in a temporary exhibition}

Resumo: As coleções provenientes de acontecimentos históricos num contexto bélico, como os campos de concentração nazistas da Segunda Guerra Mundial, têm um valor cultural e social relevante como testemunho material dos factos. Alcançar um equilíbrio igual entre sua acessibilidade social e sua conservação é o principal desafio enfrentado por museus memoriais como o Museu Estatal de Auschwitz-Birkenau, ao qual se somam todos os riscos que uma transferência para uma exposição temporária implica, especialmente aqueles riscos de origem antrópica, seja fortuitos ou intencionais. A análise crítica destes indicadores irá ajudar-nos a reconhecer as possíveis emergências e consequentes danos nas coleções de memória em trânsito e a abordar a sua conservação na perspetiva da sustentabilidade.

Palavras-chave: coleções de memória trágica, risco antrópico, exposição temporária, sustentabilidade, Museu Estatal de AuschwitzBirkenau

\section{O estudo da significância e dos indicadores de risco antrópicos para a preservação de coleções de memória trágica numa exposição temporária}

Abstract: Collections that were generated through a historical fact in a war context such as nazi concentration camp during 2 nd World War, have a relevant cultural and social value as material testimony of facts. The main challenge that face Memorial museums is to achieve an equal balance between its social accessibility and its conservation. Also, it is necessary to take into account those all added risks when those collections are on loan for a traveling exhibition, especially the intentional or casual anthropic factors. The critical analysis of the risks will allow us to recognize the possible emergency issues and the consequential damage on the collection of tragical memory when is in transit and address their conservation for a sustainable approach.

Keywords: collections of tragic memory, anthropic risk, temporary exhibition, sustainability, Auschwitz-Birkenau State Museum. 


\section{Introducción}

Las colecciones de patrimonios difíciles están directamente vinculadas a los lugares donde ocurrieron los hechos, como los campos de concentración nazi. El Museo Estatal de Auschwitz-Birkenau, está asociado al espacio histórico trágico y reconocido como un lugar de memoria. La conservación en un lugar así, centro de investigación y herramienta didáctica, consiste en la preservación, en la pervivencia de los testimonios tal cual se encontraron, poniéndolos a salvo e intentando detener los efectos del paso del tiempo a través de un plan global de conservación (Bartyzel 2021). Tanto su infraestructura como las colecciones del museo están sujetos a proyectos de conservación y a un plan de emergencia, con el objetivo de protegerlas para las generaciones presentes y las futuras (ABSM website "Master Plan"; ABSM website "Projects").

Partiendo de un proceso de investigación desde la conservación-restauración ${ }^{[1]}$ activo en la actualidad, este artículo tiene como objetivo destacar la importancia de los riesgos antrópicos de las colecciones de la memoria trágica en itinerancia, mediante el estudio cualitativo de los museos memoriales, sus retos y dificultades y el análisis crítico de los posibles daños antropogénicos externos e internos dada su relevante significación histórica, política, social y cultural. Como conclusiones, serán ordenados según las fases de gestión de una exposición temporal para su seguimiento en la praxis preventiva.

\section{Museos memoriales y su conservación}

Accesibilidad y conservación son los dos platos de la balanza en la búsqueda del equilibrio museístico, y la cesión temporal de los objetos de estas instituciones para una exposición temporal itinerante conlleva un conjunto de riesgos añadidos a los que habitualmente están expuestos en los diversos espacios públicos o privados. Su misión testimonial y didáctica los convierte en elementos que deben ser preservados para a su vez, ser mostrados y comunicados con todo su mensaje intacto y auténtico (Bornamm 2018: 38). Asimismo, su potente significado los hace ser más vulnerables y estar más expuestos a un deterioro antrópico.

La base teórica y reflexiva para construir una evaluación o marco de significancia de las colecciones de memoria trágica es todavía un proceso vivo abordado fundamentalmente desde las áreas sociales y antropológicas (Arrieta 2016; Maceira 2012; Ortiz y Sánchez 2008). Si atendemos a su caracterización y a la gestión de riesgos de esta tipología de colecciones, se debe acudir a las fuentes primarias como son los museos memoriales para investigar sobre los sistemas de documentación, catalogación y conservación, donde los diversos laboratorios generan una rica e interesante información vinculada con el desempeño del día a día, y que es de uso específico para la institución museística
(Strzelcyz 2004) [figura 1]. Se trata de unas instituciones que merecen especial atención y cuya contextualización nos ayuda a comprender sus acciones de conservación de los patrimonios difíciles (Klinger 2018; Jastrzębowski 2018; Rymaszewski 2004).

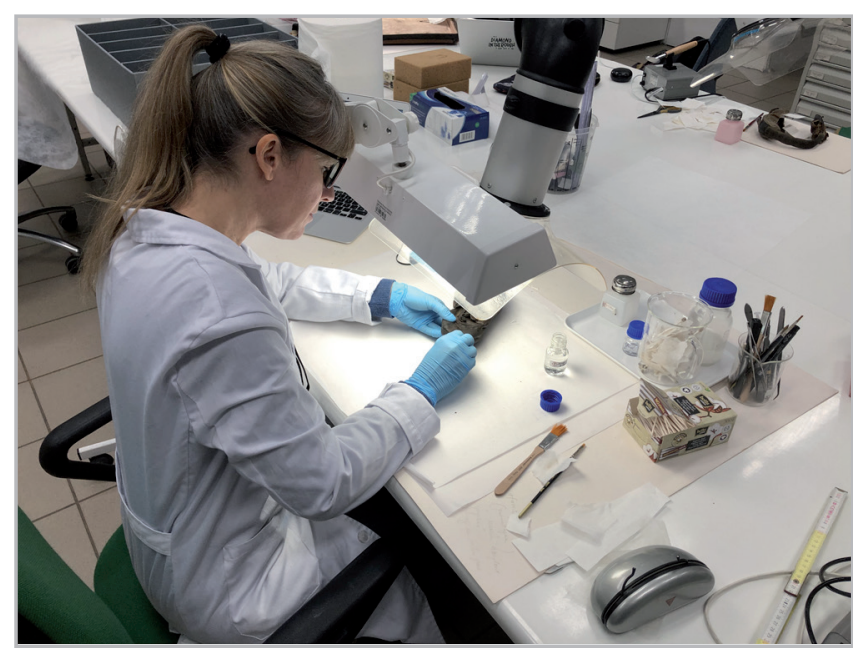

Figura 1.- . Proceso de conservación de colección de zapatos del museo en el Laboratorio del Museo Estatal de AuschwitzBirkenau. Foto: Andrzei Jastrzębowski.

Sin embargo, al identificar el contexto museístico comprobamos la multiplicidad de instituciones y museos memoriales. Reconocemos una heterogeneidad y multiplicidad de diversas fórmulas de gestión (públicos y privados) y por tanto con herramientas diversas para su registro y conservación en una exposición temporal (Galán y Vieira 2020). Así, podemos citar los museos vinculados al lugar histórico y Patrimonio de la Humanidad, como el Museo de Auschwitz-Birkenau (Polonia); los museos de cultura judía, como el Museo Judío de Oświęcim (Polonia) o el Museo Judío de Amsterdam (Holanda); los Centros de estudio como The Wiener Library (Reino Unido); archivos de herencia judía y religiosa como Amud Aish Memorial Museum de Nueva York (Estados Unidos); los museos memoriales en recuerdo de la SHOA, como Yad Vashem de Israel (Jerusalem); los museos y fundaciones creadas con la misión de recordar la historia y como lugar de encuentro comunitario con los supervivientes: United State Holocaust Museum Memorial USHMM, (Washington), Museum of Jewish Heritage; y también los museos de carácter bélico, como los museos de Historia Militar: Imperial War Museum de Londres (Reino Unido); las casas museo relacionadas con historias personales que han pasado a ser universales, como el Museo de Ana Frank de Amsterdam (Holanda).

Por otra parte, existen toda una serie de fundaciones, como Spungen Foundation (Estados Unidos), coleccionistas privados, como Miroslaw Ganobis de Oświęcim (Polonia) y más directamente los familiares y descendientes de supervivientes, como Melcher de Wind (Holanda), la profesora Judy Tylor (Israel), o la profesora Anne Ruth Lenga 
(Reino Unido), entre otros, que custodian su legado familiar y que lo han cedido para exposiciones temporales ${ }^{[2]}$.

La heterogeneidad de agentes señalada implica un gran reto como es la diversidad en el tratamiento de los objetos por sus propietarios, según procedan de institución o de organismo privado. Además, se requiere poner en práctica nuevos canales de comunicación con estos agentes, custodios de estos objetos: museos memoriales, museos diferentes, y organismos privados: fundaciones familiares y familias directas de supervivientes al Holocausto.

Dichos museos a la hora de idear planes de salvaguarda en los que se integra la gestión de riesgos deben adaptar fórmulas originadas para colecciones artísticas e históricas, a colecciones formadas por objetos procedentes de un suceso trágico como es el Holocausto, en los que la significancia de este patrimonio aborda valores intangibles específicos vinculados con la memoria y la remembranza y que además está vinculado con movimientos políticos y sociales que hicieron posible este genocidio hace 75 años, y que permanecen en la estructura social internacional como hemos podido comprobar el pasado 9 de enero de 2021 a las puertas del Museum of Jewish Heritage en Nueva York, en relación con los acontecimientos de asalto al Capitolio norteamericano en Washington (Eyewitness news 2021).

\section{Significancia de las colecciones de la memoria trágica}

Las colecciones de la memoria que custodian dichos museos, por su significancia social, política y cultural pueden verse afectadas en menor o mayor medida por estas causas de degradación cada vez que salen de su institución de origen. Por ello, para evaluar posibles riesgos, se requiere proceder a su valoración y detectar las posibilidades de que ocurra un riesgo antes de que suceda (Durán 2019). Entendemos la valoración o significancia, por tanto, como la acción de recopilación y comprensión del valor de los bienes culturales previa a todo plan de conservación preventiva y de prevención de riesgos, y en el caso de las colecciones de la memoria trágica a identificar posteriormente por sus características y significado, cómo está de expuesta a los posibles riesgos antrópicos.

La evaluación de estas colecciones a través de la retícula de NARA (Van Balen 2008) o abordada desde las diversas fuentes de evaluación de bienes culturales (Reed 2018; Russel y Winkworth 2009) proporciona el siguiente análisis crítico de los atributos, valores histórico-culturales, valores sociales y valores de uso.

Respecto a los atributos, conocer su naturaleza y valorar el análisis del estado de conservación es prioritario. Respecto a su constitución material, destaca su vulnerabilidad y fragilidad, intrínsecamente relacionadas con su constitución material, al momento y lugar en el que se crearon: no duraderos, rápido y a escondidas, reciclados, con materiales de deshecho. Esto supone que los museos estudian caso a caso las posibilidades de su cesión temporal y establecen sus requerimientos de manera que puedan manipularse con mayores precauciones que otro tipo de materiales (Klinger 2018). Además, hay que tener en cuenta que en una exposición temporal que itinera la manipulación de los objetos se reproduce de manera sistemática en breves periodos de tiempo y que a menudo se resuelve con su sustitución. Estas colecciones vulnerables, además, se ubican en una exposición temporal en una ubicación externa a la que el objeto está habituado (bien en sala de reservas, bien en la exposición permanente de su institución de origen).

Respecto a la procedencia, la documentación que atestigua el origen es un punto fuerte considerando el mercado negro en torno a la II Guerra Mundial y el nazismo; sobre su rareza y representatividad, ciertamente difícil de evaluar cuando cada objeto personal es único y cuenta una historia individual de supervivencia. Para el Museo de AuschwitzBirkenau este punto es muy importante a la hora de desarrollar los planes de salvaguarda, y la valoración de sus colecciones es compleja y profunda. Tratarse de un elemento único y representativo lo sitúa como un elemento prioritario para su rescate (Sawicki et al. 2015). Atendiendo al valor de conjunto que en nuestro caso de estudio es muy importante. La ingente acumulación de enseres cotidianos, cepillos, maletas, etc. nos habla en su conjunto de la magnitud del Holocausto [figura 2]. Es por ello por lo que a menudo se han presentado museografías acumulativas como las exposiciones actuales en el Museo Estatal de Auschwitz-Birkenau, aunque la tendencia actual en las nuevas museologías y museografías proyectadas por el museo citado es seleccionar ciertos objetos y custodiar en la sala de reservas la mayor parte del conjunto para preservar y prevenir riesgos por manipulación (Sawicki et al. 2015). En este caso, dañar el objeto es dañar el valor del conjunto total.

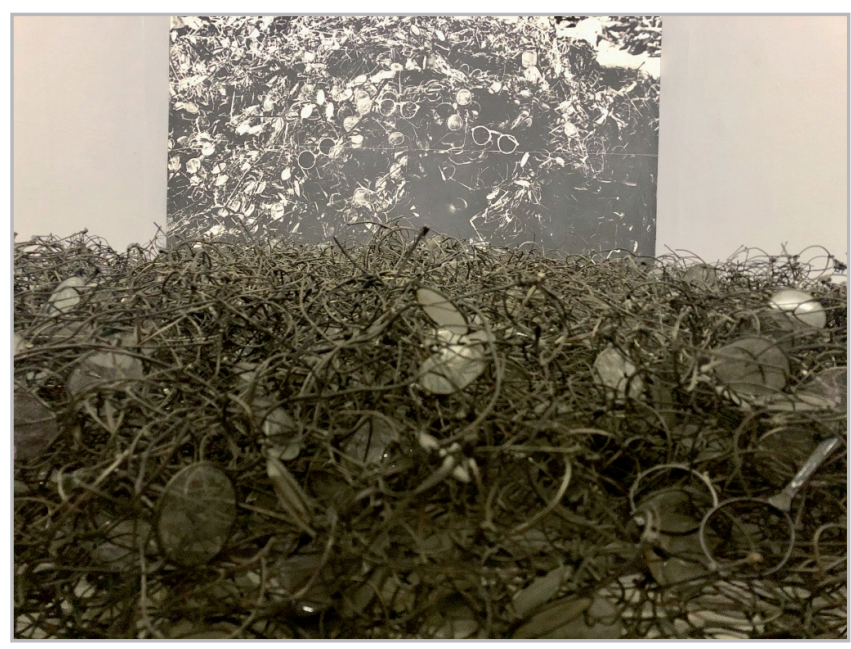

Figura 2.- Vitrina con gafas presentadas siguiendo la museografía acumulativa por el significado de conjunto en la exposición permanente del Museo Estatal de Auschwitz-Birkenau. Foto: Ana Galán 
Respecto a los valores histórico-culturales, las colecciones de la memoria pueden ser valoradas por su vinculación a los lugares de importancia histórica donde se cometieron los crímenes, por la vinculación con una persona concreta o un grupo. Cuestión bien difícil cuando se evalúan las colecciones para un plan de seguridad de una exposición temporal: ¿qué peso histórico cultural es mayor, el del escritorio original de Rudolf Höss, comandante del campo de concentración de Auschwitz, o la manivela que abría la estantería tras la que se escondía la familia Frank en su domicilio de Ámsterdam? [figura 3]. Complejidad que se extiende a la evaluación de valores artísticos, puesto que la expresión pictórica, narrativa y artesana estuvo presente principalmente en los elementos creados en los campos. Por tanto, evaluar el diseño, concepto, técnica, marca o creatividad de entre todos los objetos producidos por los prisioneros supone necesariamente la valoración conjunta de otros factores. También tenemos el caso de obra de artistas que ya desempeñaban su carrera profesional como tales o que florecieron después, y que crearon obra directamente relacionada con su experiencia trágica, como el artista David Oleré (Polonia, 1902-1985) o la pintora Ceija Stojka (Austria, 1933-2013) (MNCARS 2020). Por otra parte, todos y cada uno de los objetos que tratamos recogen entre sus valores el ser testigos del ataque a los derechos fundamentales. Los museos que los custodian reconocen el gran valor de información que contienen como testimonio material y aseguran que su conservación es vital como documento, pues de alguna manera ayudarán a mantener la memoria de los hechos una vez los últimos supervivientes desaparezcan. La dificultad radica en determinar entre todos los objetos, cuál es la jerarquía o selección del más importante al menos importante, cuando todos son potencialmente "objeto con fines de estudio, investigación, ciencia, documentación, referencia o archivo" (Durán 2019).

Y llegamos a los valores sociales, en los que evaluamos el significado de las colecciones de la memoria para un grupo de personas o una comunidad por razones políticas, sociales

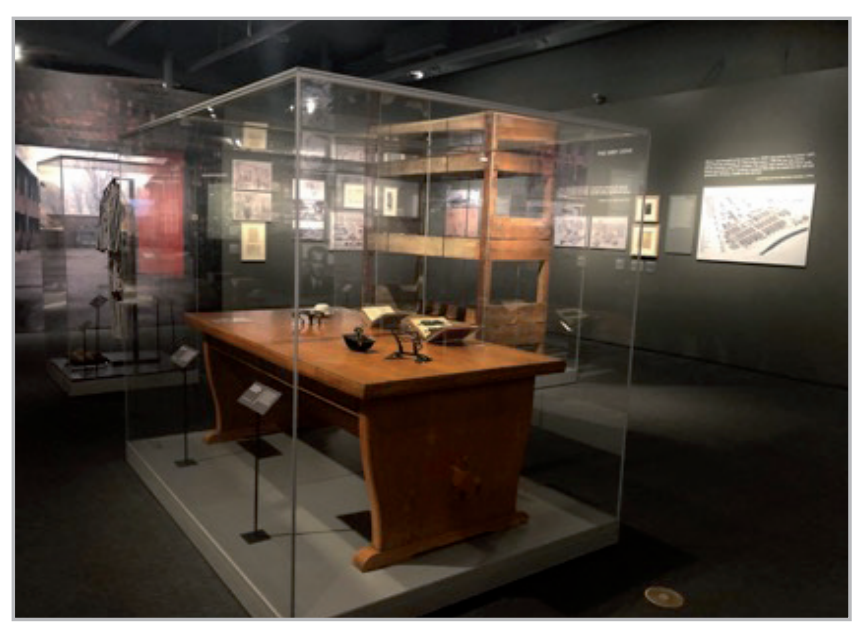

Figura 3.- Vitrina de la exposición temporal "Auschwitz. Not Long Ago. Not far away" con el escritorio original de Rudolf Höss, colección del Museo Estatal de Auschwitz-Birkenau. Foto: Ana Galán. y religiosas. $Y$ este es sin duda otro rasgo significativo de los objetos del Holocausto como hemos avanzado en la introducción. El motivo de su salvaguarda es precisamente porque son importantes para la identidad de las comunidades que fueron víctima de los crímenes públicos, pero, también para toda la sociedad. Por otra parte, son objetos de la memoria, y como tal evocan una remembranza, una experiencia revivida intensa (Cywiński 2015).

Muy relacionado con los valores sociales, evaluamos los valores de uso. Tal y como comentábamos previamente, es precisamente el reconocimiento de uso lo que nos genera la necesidad de analizar los indicadores de riesgo antropogénicos. Bien para ser expuestos en un museo o en una exposición temporal, con fines didácticos y pedagógicos estas colecciones tienen un uso específico que es el de la construcción de democracias y la defensa de los derechos humanos (Maceira 2012).

\section{Riesgos antrópicos externos e internos}

Analizada su significancia, entre los riesgos que pueden afectar al patrimonio cultural, los agentes de origen humano pueden provocar un deterioro en toda su magnitud, siendo tanto externos, con intencionalidad de daño o destrucción, como internos, es decir, aquellos que afectan a las propias dinámicas sugeridas por la puesta en valor del objeto e internas al trabajo de sus profesionales (ICCROM 2001; Hekman 2010).

Cuando nos referimos a riesgos antrópicos, estamos hablando de la posibilidad de daños o pérdidas que pueden producirse en los objetos memoriales, provocados específicamente por personas. En su identificación, es relevante conocer la vulnerabilidad como un factor intrínseco a los objetos de la memoria trágica expuestos a sufrir el daño (infraestructura, personal, colecciones), así como la peligrosidad, un factor de riesgo externo, considerada como la probabilidad de ocurrencia de un hecho potencialmente dañino durante cierto periodo de tiempo, en un lugar concreto (Herráez, Durán y Pastor 2019).

Por tanto, es la propia naturaleza de estas colecciones y su estado de conservación el factor intrínseco que determina su vulnerabilidad, pero también la logística o espacios en los que se va a ubicar en un momento $u$ en otro cuando se cede en préstamo, así como la participación del diverso personal y su preparación que atenderá las necesidades de dichos objetos. Respecto a la peligrosidad, hay factores externos que afectan directamente a las colecciones de la memoria trágica en relación con el contexto social, político y cultural de las localidades donde se ubica la sede.

Sin embargo, no todos los daños antrópicos tienen el mismo origen, distinguiendo entre externos e internos, accidentales y provocados (Carrión 2015; Culubret et al. 2008). 


\section{—Riesgos antrópicos externos}

- Por una parte, distinguimos los daños provocados, como sabotaje, vandalismo, terrorismo, situaciones bélicas o conflictos sociales (Tremain 2009). Recientemente hemos sido testigos en los acontecimientos del asalto al Capitolio el pasado 9 de enero de 2021 de la performance ejecutada y compartida en redes sociales por grupos neofascistas por la que se tomaron fotos saludando con la bandera confederada americana a las puertas del Jewish Museum of Heritage en Nueva York, sede actual de la exposición "Auschwitz. No hace mucho. No muy lejos" y donde se custodia el vagón de la Deutsche Reichsbahn (Outlive Them NYC 2021). Este grupo de riesgos está intrínsecamente relacionado con la situación política y social de las comunidades locales.

- Los daños provocados por la sustracción o robo para el mercado negro de objetos relacionados con la II Guerra Mundial. Así ha ocurrido en el año 2009 con el robo de la señal de entrada al campo de Auschwitz (Ogrodzki 2020: 171) o la misma señal en el campo de Dachau "Arbeit Macht Frei" en el año 2017 (Blakemore 2017; The Guardian 2009) [Figura 4].

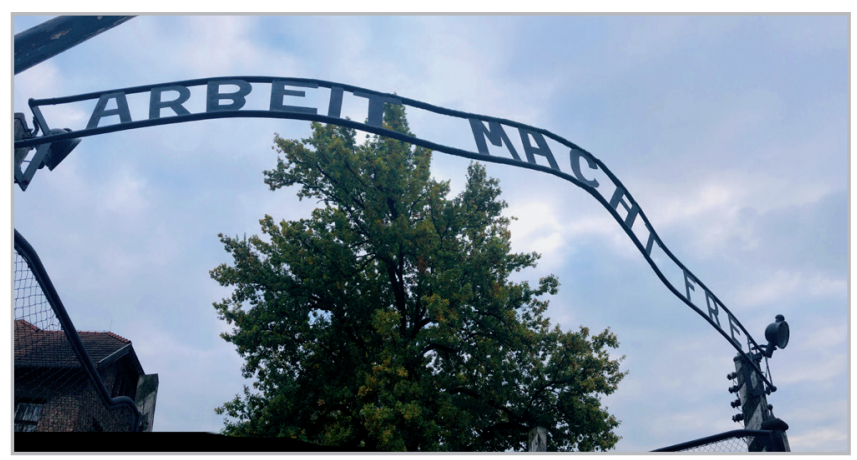

Figura 4.- Señal de acceso al campo de concentración de Auschwitz-Birkenau. En la actualidad se trata de una reproducción tras el intento de robo en 2009. La señal original se restauró y se conserva en las salas de reserva del propio museo. Foto: Ana Galán.

- Daños vandálicos generados por los visitantes, como marcas, arañazos o sustracción de elementos de las maquetas didácticas en las exposiciones temporales.

- El daño que se puede producir por la solicitud de requerimiento privado o confiscación de un objeto expuesto y por el que se pone en funcionamiento entre la institución y el país de recepción de la exposición el certificado de no confiscación, o Non seizure certificate (Woudenberg 2010; Matassa 2014; Leeuw 2005). A través de esta herramienta, el país de recepción se compromete a proteger los objetos de la colección ante una demanda de propiedad que pudiera llegar a darse. En caso contrario, el objeto es confiscado y custodiado en almacenes no habilitados para la conservación del patrimonio hasta que se dirime el juicio (Peterson et al. 2010).

\section{— Riesgos antrópicos internos}

- Los daños fortuitos no intencionados, como la disociación. Por su gran carga histórica y dramática se trata de un tipo de patrimonio difícil, sensible, requiriendo un tratamiento narrativo cuidadoso y una manipulación ajustada a su significancia. $Y$ esta dificultad también se produce en los tratamientos de documentación, pues si bien se utilizan herramientas preparadas para abordar el patrimonio histórico-artístico en el contexto de una exposición temporal, existen ciertas lagunas para abordar el patrimonio de la memoria trágica, dado su fuerte carácter antropológico y social, como ya hemos comentado previamente. Además, comprobamos la ausencia de tesauros, por lo que no todos los museos nombran de la misma manera objetos de similar naturaleza o procedencia favoreciendo el factor antrópico de deterioro que es la disociación (Galán 2020).

- Daños fortuitos no intencionados derivados del uso del objeto una vez expuesto. $Y$ es que, por su significado, estos objetos custodiados por los museos y también por los familiares, pueden desempeñar su misión patrimonial y accesible de manera más activa y al ser utilizados, más allá que limitarse a la contemplación en una vitrina de una exposición temporal por parte de sus visitantes. Son objetos en uso, como el Shofar colección de Judy Tydor (Israel), instrumento litúrgico musical que fue tocado en Auschwitz y que se expone en el Museum of Jewish Heritage de Nueva York [figuras 5, 6 y 7]. De manera periódica es solicitado para ser utilizado en las festividades judías de las sinagogas de la ciudad. Por tanto, son elementos musealizados pero a la vez en uso. Forman parte de la exposición, y también de la dinámica religiosa de la comunidad judía de la ciudad. Así, abandonan puntalmente su vitrina para ser más accesibles para las comunidades, materializando lo que en múltiples convenios y cartas internacionales atestiguan, y que supone reconocer el gran peso que tiene la sociedad para reconocer los valores específicos con los cuales se identifica (Galán 2020).

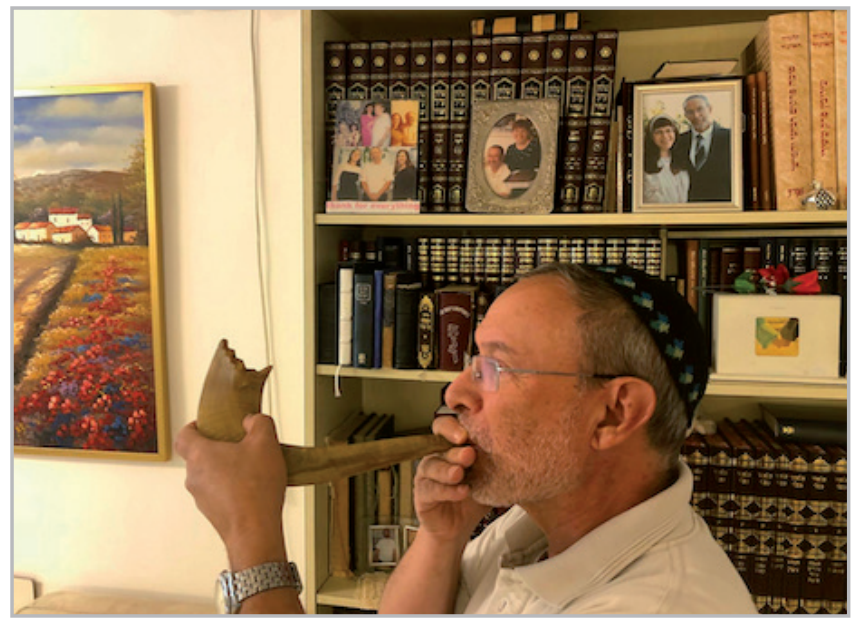

Figura 5.- Familiar de superviviente haciendo sonar el Shofar o instrumento litúrgico judío antes de su embalaje y traslado a la exposición temporal. Foto: Judy Tydor (Baumel) Schwartz. 


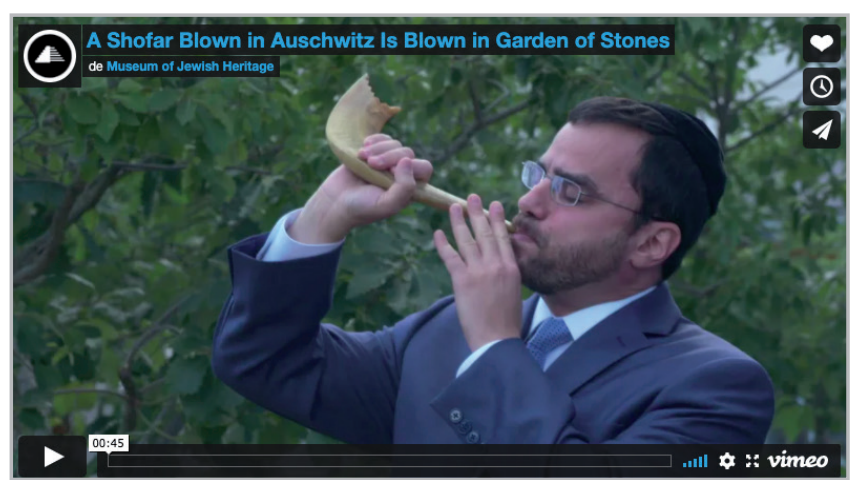

Figura 6.- El Shofar de la familia Tydor siendo sonado en celebración religiosa judía en el Jewish Museum of Heritage, de Nueva York. Foto: Museum of Jewish Heritage, de su canal de Vimeo

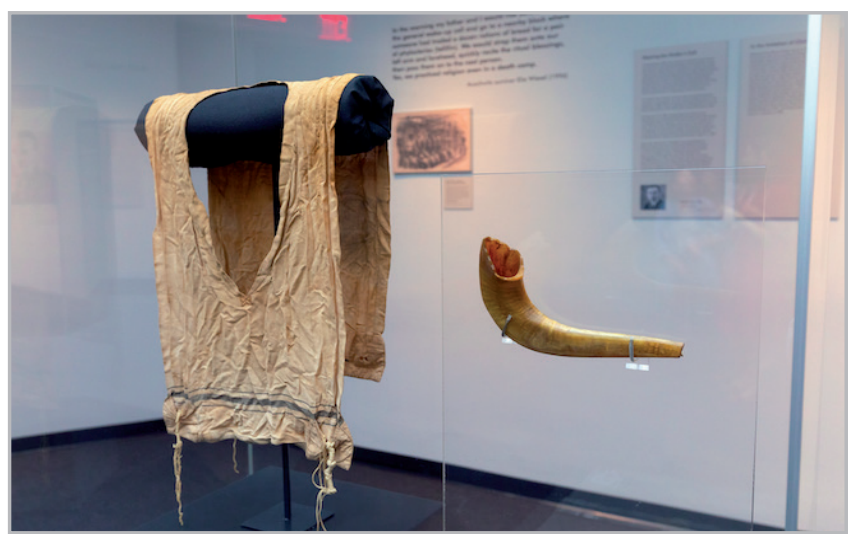

Figura 7.- El Shofar de la familia Tydor en la vitrina de la exposición "Auschwitz. Not Long Ago. Not far away" en el Jewish Heritage Museum de Nueva York, en los momentos en los que no es utilizado. Foto: Ana Galán.

- Los daños fortuitos no intencionados derivados de la falta de mantenimiento en las instalaciones o ausencia de estándares mínimos para la conservación de colecciones específicas, y cuya herramienta de control es el informe de condiciones o Conditions Report. También es relevante que, para evitar estos daños, dicha institución conozca las características de las colecciones y de la exposición, y sus necesidades para preservarlas correctamente antes, durante y después de la producción de la misma. Espacio, teniendo en cuenta las seis capas de protección o envoltorio: la región, el lugar, el edificio, la sala, el mobiliario receptorio-vitrina y el soporte (Michalski y Pedersoli 2016).

- Los daños fortuitos no intencionados, como los descuidos derivados de la ausencia de una protocolización de acciones sobre las colecciones y de mantenimiento en la exposición que también se relaciona con la visita y que afecta al personal o a los agentes sociales, culturales y gestores involucrados (Michalski, Pedersoli y Antomarchi 2016). Este grupo de riesgos afectaría sobre todo a la dinámica de la exposición temporal.

El equipo y sus procedimientos son un pilar de estudio básico y en el que nos integramos para comunicar y tomar decisiones de manera conjunta. Desde el equipo de gestión, coordinación y toma de decisiones, el equipo científico y la selección de piezas que formarán parte de la exposición, el equipo de diseño que lo distribuirá en el espacio y el equipo de producción que preparará el contexto adecuado para que las colecciones comuniquen apropiadamente al visitante con todas las garantías de instalación y prevención de riesgos durante y después del montaje (Biedermann, Ferreiro y Galán 2018).

Estas tipologías de riesgos antrópicos engloban las diversas casuísticas y posibilidades catastróficas cuyo resultado sea el daño o pérdida de los objetos del patrimonio memorial cedidos en préstamo temporal, que salen de sus instituciones de origen, se trasladan y forman parte de una exposición accesible para la sociedad de recepción, en la nueva sede donde permanecerá temporalmente.

\section{Conclusiones}

Reconocer las causas humanas que pueden afectar a las colecciones de la memoria trágica, bien externas al proyecto expositivo bien internas al mismo por las que se puede provocar un daño o pérdida, son clave para elaborar un plan de emergencias además de introducirlo como protocolo habitual de conservación preventiva en una exposición temporal (Maxwell et al. 2018) [Figura 8]. Es decir, en las tres fases en las que se gestionan las colecciones, gestación, organización y desarrollo: programación y producción, montaje e instalación, periodo expositivo, desmontaje y dispersión o reorganización en el caso de que además sea itinerante (Fernández et al. 2008), y en las diversas tareas de documentación, registro y logística, manipulación y transporte e instalación y seguimiento del estado de conservación (Galán, Biedermann y Ferreiro 2020).

Por otra parte, a la hora de identificar el contexto y los distintos agentes que intervienen en la toma de decisiones sobre las colecciones o sobre aspectos que pueden afectarles, podemos señalar: por una parte, la heterogeneidad en origen de los propietarios de las colecciones o la institución de origen, público o privada, familia o fundación; la sede de la exposición: museo o institución de llegada, personal propio (gestión, mantenimiento, limpieza, seguridad, etc.); las comunidades, especialmente la sociedad local y cómo participa en la exposición; y finalmente el equipo organizador: comisarios, gestores, diseño-museografía, producción, etc. Los procedimientos y herramientas dependen de los agentes que participan en la exposición temporal.

La conservación y la accesibilidad en las colecciones de la memoria van de la mano y son uno de los principales retos de estos museos y de sus proyectos expositivos. Facilitar la movilidad de los objetos también supone una serie de riesgos antrópicos, principalmente por su uso social, por su significado político y riesgo de vandalismo, y por su vulnerabilidad a la hora de manipularlo para su embalaje e instalación. 


\section{FASE | Preparación}

- Ausencia de un análisis y recopilación de la documentación pormenorizada de los distintos objetos y sus requerimientos especificos como colecciones de memoria.

- Diversa denominación o denominación no coincidente entre los diversos agentes implicados. Ausencia de Tesauro.

- Ausencia de un cronograma de registro y movimiento de las colecciones acorde con la dinámica de sus propietarios.

- Ausencia de protocolos de comunicación e intercambio de información entre los agentes implicados.

- Ausencia de aproximación comunicadora a las familias con objetos de la memoria.

- Ausencia de estándares de manipulación especificos.

- Ausencia de previsión de seguridad; ausencia de solicitud de los requerimientos legales como el Certificado de no confiscación de objetos del Holocausto, entre otros.

\section{FASE IV operaciones}

- Multiplicidad de puntos de salida y de retorno de las colecciones.

- Ausencia de protocolos claros de ejecución del registro, salida y llegada de los objetos de la memoria de la institución prestataria a la sede receptora

- Posibilidad de solapamiento de llegada y atención de los objetos por el momento en el que materializa la cesión de los propietarios.

- Ausencia de protocolos de instalación de los objetos de la memoria.

- Ausencia o desconocimiento de los de estándares de calidad de las empresas locales subcontratadas en cada ciudad donde la exposición itinera.

- Errores técnicos.

- Ausencia de formación en el personal de instalación de las colecciones, entre otros.

- Además de estos riesgos por ausencia, cabe integrar aquellos que se generen de manera fortuita como accidentes, o provocados como intentos de robo en el trayecto, siendo necesaria escolta acompañando el recorrido de las piezas.

\section{FASE II Operaciones}

- Multiplicidad de puntos de salida y de retorno de las colecciones.

- Ausencia de protocolos claros de ejecución del registro, salida y llegada de los objetos de la memoria de la institución prestataria a la sede receptora

- Posibilidad de solapamiento de llegada y atención de los objetos por el momento en el que materializa la cesión de los propietarios.

- Ausencia de protocolos de instalación de los objetos de la memoria.

- Ausencia o desconocimiento de los de estándares de calidad de las empresas locales subcontratadas en cada ciudad donde la exposición itinera.

- Errores técnicos.

- Ausencia de formación sobre las colecciones del personal de instalación.

- Además de estos riesgos por ausencia, cabe integrar aquellos que se generen de manera fortuita como accidentes, o provocados como intentos de robo en el trayecto, siendo necesaria escolta acompañando el recorrido de las piezas.

\section{FASE III Exposición}
- Impacto de la significancia de las colecciones y solicitud de uso cultural o religioso fuera de su vitrina.
- Ausencia de seguridad, cámaras de vigilancia, personal.
- Errores técnicos.
- Posibilidad de actos vandálicos, hechos terroristas, sustracción o robo de elementos de la exposición.
- Requerimiento de confiscación por iniciativa privada.
- Ausencia de seguimiento de las condiciones de las colecciones.
- Ausencia de los requerimientos de conservación preventiva para las colecciones de la memoria.
- Impacto social y uso del espacio expositivo para ceremonias, reuniones, encuentros propiciados por las comunidades.
- Impacto de la afluencia de visitantes en el recorrido propuesto y ubicación de las vitrinas.

Figura 8.- Gráfico de riesgos antrópicos de las colecciones de la memoria en una exposición temporal. Foto: Eduarda Vieira y Ana Galán.

En conclusión, desde el análisis de las colecciones de la memoria, pasando por las fórmulas de trabajo de los museos prestadores, las sedes de recepción, así como las comunidades que directa o indirectamente están relacionadas con el patrimonio del Holocausto, el equipo degestión de una exposición temporal tiene ante síun reto para salvaguardar y proteger el patrimonio de los posibles riesgos antrópicos que pudieran llegar a producirse, teniendo en cuenta que el estudio de los indicadores de riesgos antrópicos integra no solo los descuidos o mala praxis, tanto fortuitos como intencionados, sino también la ausencia de una previsión en cada una de las fases en las que las colecciones de la memoria son gestionadas en las exposiciones temporales itinerantes. Gestionar los riesgos supone avanzar hacia unos entornos museísticos más resilientes y sostenibles (McGhie 2020).

\section{Notas}

[1] El estudio de la preservación de los patrimonios difíciles en tránsito es uno de los objetivos de investigación postdoctoral desarrollado en la actualidad por las autoras en CITAR, Centro de Investigación en Ciencia y Tecnología de las Artes, de la Escuela de las Artes de la Universidad Católica Portuguesa (Citar-EA-UCP, Oporto).

[2] La exposición temporal itinerante "Auschwitz. Not Long Ago. Not Far away", es un proyecto de cooperación entre la empresa Musealia y el museo estatal de Auschwitz-Birkenau de Polonia, punto de referencia y objeto de nuestro estudio, así como el propio museo. 


\section{Referencias}

ABSM, AUSCHWIZT-BIRKENAU STATE MUSEUM Website. "Master Plan for Preservation. Preservation Area". http://auschwitz.org/en/ museum/preservation/master-plan-for-preservation [consulta: 10/11/2020].

ABSM, AUSCHWIZT-BIRKENAU STATE MUSEUM Website. "Projects" http://auschwitz.org/en/museum/preservation/projects [consulta: 10/11/2020].

ARRIETA URIZBEREA, I. (ed.) (2016). Lugares de memoria traumática. Representaciones museográficas de conflictos políticos y armados. Bilbao: Universidad del País Vasco.

BARTYZEL, B. (2021)."Auschwitz: Significado y simbología", en Mes del Holocausto, Centro Sefarad-Israel Canal de YouTube https:// www.youtube.com/watch?v=NXI6OzriPTM\&feature $=$ emb logo [Consulta: 30/01/2021]

BIEDERMANN, A., FERREIRO, L. y GALÁN-PÉREZ, A. (2018). "Auschwitz -una exposición itinerante- diseño, gestión de colecciones y de información". En ReVisiones sobre Arte, patrimonio y tecnología en la era digital. Foradada Baldellou, Irala-Hortal (eds.). Zaragoza: Gobierno de Aragón.

BLAKEMORE, E. (2017). "Stolen 'Arbeit Macht Frei' Gate Returns to Dachau. The identity of the thieves remains a mystery", en SMITHSONIAN Magazine. https://www.smithsonianmag. $\mathrm{com} / \mathrm{smart}$-news/stolen-arbeit-macht-frei-gate-returnsdachau-180962252/ [consulta: 2/01/2021]

BORNAMM, M. (2018). "In direct contact. material testimonies from the Auschwitz Concentration Camp. The conservators perspective", Memory of the Holocaust, MOCAK Forum Special Edition 1/2018: 38-43.

CARRIÓN GÚTIEZ, A. (coord.) (2015). Plan Nacional de Emergencias y Gestión de Riesgos en Patrimonio Cultural. Madrid: Ministerio de Educación, Cultura y Deporte.

CULUBRET WORMS, B., HERNÁNDEZ AZCUTIA, M., HIDALGO CÁMARA, E., MARTÍNEZ DE MARAÑÓN YANGUAS, M. y RALLO GRUSS, C. (2008). Guía para un Plan de protección de colecciones ante emergencias. Madrid: Ministerio de Cultura.

CYWINSKI, P. (2015). Epitaph. Oswiecim (Polonia): AuschwitzBirkenau State Museum.

DURÁN ROMERO, D. (2019). “Evaluación de Bienes culturales", en Seminario Significancia. Ministerio de Cultura y Deporte, Instituto de Patrimonio Cultural de España. https://www.youtube.com/ watch?time continue $=7 \& \mathrm{v}=\mathrm{M} 3 \mathrm{aflH} 3 \mathrm{Dpw} \&$ feature $=\mathrm{emb}$ logo [Consulta: 05/11/2019]

EYEWITNESS NEWS (2021). "Confederate flag found tied to door of Museum of Jewish Heritage". https://abc7ny.com/ museum-of-jewish-heritage-confederate-flag-anti-semitismsemitic/9472755/ [Consulta: 30/01/2021]
FERNÁNDEZ, C., ARECHAVALA, F., MUÑOZ-CAMPOS, P., TAPOL, B.D. y DE SORIA, F. D. (2008). Conservación preventiva y procedimientos en exposiciones temporales. Madrid: Grupo Español de IIC y Fundación Duques de Soria.

GALÁN-PÉREZ, A., BIEDERMANN, A., FERREIRO, L., et. al. (2020) "Preventive conservation and sustainability of heritage collections in transit", Estudos de Conservação e Restauro, 11: 31-51. https:// doi.org/10.34632/ecr.2020.9586.

GALÁN-PÉREZ, A., (2020). "Significancia del património trágico. Hacia nuevos paradigmas de conservación preventiva", UNICUM, 19: $59-70$.

GALÁN-PÉREZ, A. y VIEIRA, E. (2020). "Museos memoriales, museos diferentes: buscando claves para su conservación", Diferents. Revista de museus, 5: 36-55. https://doi.org/10.6035/ Diferents.2020.5.3.

HEKMAN, W. (ed.) (2010). Handbook on Emergency procedures. Amsterdam: ICOM, International Committee for Museum Security.

HERRÁEZ, J. A., DURÁN, D. y PASTOR, M. J. (coord) (2019). Guía para la Elaboración e Implantación de Planes de Conservación Preventiva. Madrid: Ministerio de Cultura y Deporte.

ICCROM (2001). "The causes of deterioration of cultural heritage. Graphic. Gaël de Guichen", en Preventive conservation. https:// www.iccrom.org/section/preventive-conservation [consulta: 2/01/2021].

JASTRZĘBIOWSKI, A. (2018). "Conservation treatment of metal artefacts from the collections of the Auschwitz-Birkenau State Museum in Oświęcim". En Conservator's Conference: Renovated, rebuilt, saved - how they survived WWII. Conservation, preservation and prevention of items from the years 1939-1945. WARSAW RAISING MUSEUM, 12-13 APRIL 2018 WARSAW, 42-43.

KLINGER, J.E. (2018). "Criteria for the Conservation of Holocaust Materials" En Conservator's Conference: Renovated, rebuilt, saved how they survived WWII. Conservation, preservation and prevention of items from the years 1939-1945. Warsaw (Polonia): Warsaw Raising Museum, 12-13.

LEEUW, R. (ed.) (2005). "Immunity from seizure". En Lending to Europe Recommendations on collection mobility for European museums. European Commission Group of Experts, Council Resolution 13839/04. https://www.muziejai.lt/ImagesNew/ LENDINGTOEUROPE PDF 051105.pdf [consulta: 2/01/2021].

MACEIRA OCHOA, L. (2012). Museo, memoria y derechos humanos: itinerarios para su visita. Bilbao: Deusto Publicaciones. Cuaderno Deusto de Derechos Humanos № 68.

MATASSA, F. (2014). "Applying for Immunity from Seizure for International Loans In". En ICOM UK Resources website. https:// uk.icom.museum/resources/guidance-articles/applying-forimmunity-from-seizure-for-international-loans-in/ [consulta: 2/01/2021] 
MICHALSKI, S. \& PEDERSOLI, J. L. (2016). The ABC Method: a risk management approach to the preservation of cultural heritage. Ottawa: Canadian Conservation Institute - ICCROM.

MICHALSKI, S., PEDERSOLI, J. L. \& ANTOMARCHI, C. (2016). A guide to Risk Management of cultural Heritage. Risk Management. Ottawa: Canadian Conservation Institute - ICCROM.

MAXWELL, I., MILOS, D., VINTZILEOU, E., BONAZZA, A. \& HANUS, C. (2018). Safeguarding cultural heritage from natural and man-made disasters. A comparative analysis of risk management in the EU. Brussels: European Commission. https://doi.org/0.2766/224310

McGHIE, H. A. (2020). Museums and Disaster Risk Reduction: building resilience in museums, society and nature. Curating Tomorrow, UK. https://www.hands-on-international.net/wp-content/uploads/ museums-and-disaster-risk-reduction-2020.pdf [consulta: 15/01/2021].

MUSEO CENTRO DE ARTE REINA SOFÍA, MNCARS (2020). "Ceija Stojka. Esto ha pasado". Exposición temporal, 22 noviembre, 2019 - 1 junio, 2020. https://www.museoreinasofia.es/exposiciones/ ceija-stojka [consulta: 15/01/2021].

OGRODZKI, P. (ed) (2020). "Museums Are Always in Danger. Theft at the Auschwitz-Birkenau Museum". En Safe Museums, Safe Collections, Ogrodzki, P. (ed). Warszawa: Narodowy Instytut Muzealnictwa i Ochrony Zbiorów, 179-182.

ORTIZ GARCÍA, C. y SÁNCHEZ CARRETERO, C. (2008). "Archivos etnográficos, memoria y nuevos patrimonios: el caso del archivo del duelo". En Patrimonios culturales: Educación e interpretación. Cruzando límites y produciendo alternativas, Pereiro, X., Prado, S. y Takenaka, H. (coords). Donostia: Ankulegi, 155-170.

OUTLIVE THEM NYC (2021). C/w antisemitism. Local Nazi Jovi Val bragged on Telegram about sieg heiling outside the Museum of Jewish Heritage last week, the very same night a confederate flag was tied to its front door. He's seen here holding a fascist'American Falangist Party'flag with 2 other people. @outlivethemnyc https:// twitter.com/outlivethemnyc/status/1349421384913575936 [consulta: 30/01/2021]

PETERSON, S., HAGEDORN-SAUPE, M., TEIJAMARI, A. \& WEIJ, A. (eds.) (2010). Encouraging collections mobility. A way forward for Museums in Europe. Helsinki: Finnish National Gallery.

REED, C. (2018). Reviewing significance 3.0, a framework for assessing museum collections' significance, management and use. https://collectionstrust.org.uk/resource/reviewingsignificance-3-0/ [consulta: 30/01/2021]

RUSSEL, R. \& WINKWORTH, K. (2009). Significance 2.0, A guide to assessing the significance of collections, Collections Council of Australia. https://www.arts.gov.au/sites/default/files/significance2.0.pdf?acsf files redirect [consulta: 15/01/2021]

RYMASZEWSKI, B. (2004). "The limits of intervention in museum and conservation practice at the Auschwitz Memorial and Museum".
En Preserving for the future. Material from an International Preservation Conference, June 23-25, 2003, Oswiecim (Polonia): Auschwitz-Birkenau State Museum, 24-34.

SAWICKI, P., BARTYZEL, B., JASTREBIOWSKA, N., OBOZA-ZIARKO, K. \& PINDERSKA-LECH, J. (eds.) (2015). To Preserve Authenticity. Nine tasks for the years 2012-2015. Oswiecim (Polonia): Auschwitz-Birkenau State Museum.

STRZELCZYK, A. (2004). “Using modern preservation workshops to protect the collections of the Auschwitz-Birkenau State Museum". En Preserving for the future. Material from an International Preservation Conference, June 23-25, 2003. Oswiecim (Polonia): Auschwitz-Birkenau State Museum, 35-37.

THE GUARDIAN (2009). Poland declares state of emergency after 'Arbeit Macht Frei' stolen from Auschwitz. https://www. theguardian.com/world/2009/dec/18/auschwitz-arbeit-machtfrei-sign [consulta: 2/01/2021]

TREMAIN, D. (2009). Robos y vandalismo. Canadian Conservation Institute - ICCROM. https://www.cncr.gob.cl/611/ articles-56474 recurso 2.pdf [consulta: 2/01/2021]

VAN BALEN, K. (2008). "The Nara Grid: An Evaluation Scheme Based on the Nara Document on Authenticity", APT bulletin, 49(2): 39-45.

WOUDENBERG, N. (2010). "Immunity from seizure: a legal exploration". En Encouraging Collections Mobility. A Way Forward for Museums in Europe, Peterson, S., Hagedorn-Saupe, M., Teijamari, A. \& Weij, A. (eds.). Helsinki: Finnish National Gallery, 184-196.

\section{Autor/es}

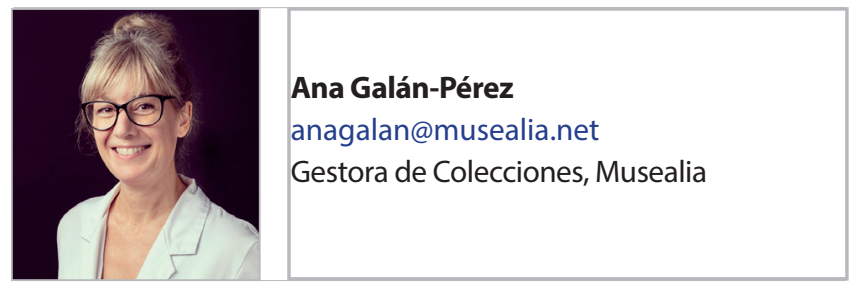

Ana Galán-Pérez, es Gestora de Colecciones en la Empresa Musealia y Profesora asociada de la Universidad Complutense de Madrid, Facultad de Bellas Artes, Dep. de Pintura y ConservaciónRestauración.

Doctora en Bellas Artes por la Universidad de Sevilla (2011), titulada en Conservación-Restauración de Bienes Culturales por la Escola Superior de Conservació i Restauració de Béns Culturals de Catalunya (2002) y Licenciada en Historia del Arte por la Universidad de Zaragoza (1998). Desarrolla su actividad profesional en el ámbito privado en la gestión de colecciones y conservación preventiva. Investigadora postdoctoral integrada en CITAR, Centro de Investigação em Ciência e Tecnologia das Artes de Universidad Católica de Porto. ORCID: 0000-0002-4583-3108 


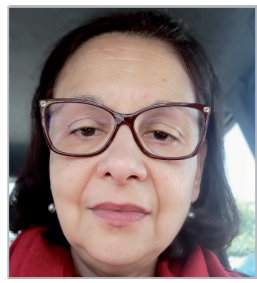

\section{Eduarda Vieira}

evieira@porto.ucp.pt

Escola das Artes de la Universidad Católica portuguesa

Eduarda Vieira es Doctora en Conservación y Restauración de Patrimonio Histórico y Artístico por la Universidad Politécnica de Valencia, y Máster en Conservación Arquitectónica por la Universidad de Évora. Actualmente es Profesora adjunta en la Escola das Artes de la Universidad Católica portuguesa (Conservación de Materiales Inorgánicos), donde coordina el programa de Doctorado en Conservación y Restauración de Patrimonio Cultural. Es Directora del Centro de Investigação em Ciência e Tecnologia das Artes (CITAR) y editora de la revista Studies in Conservation and Restoration -ECR-Estudos de Conservação e Restauro. También es miembro de ICOMOS e ICOM, e investigadora de numerosos proyectos relacionados con Conservación preventiva y Green Conservation, además de supervisora de numerosas tesis Doctorales y de Master. ORCID: 0000-0002-0620-080X

\section{(@) $\Theta \Theta$}

https://doi.org/10.37558/gec.v19i.1012 